\title{
Supplemental material: Comparing modelled fire dynamics with charcoal records for the Holocene
}

T. Brücher ${ }^{1}$, V. Brovkin ${ }^{1}$, S. Kloster ${ }^{1}$, J. R. Marlon ${ }^{2}$, and M. J. Power ${ }^{3}$

${ }^{1}$ Max Planck Institute for Meteorology, Hamburg, Germany

${ }^{2}$ School of Forestry and Environmental Studies, Yale University, New Haven, CT

${ }^{3}$ Natural History Museum of Utah, Department of Geography, University of Utah, Salt Lake City, UT 84112

Correspondence to: Tim Brücher

(tim.bruecher@mpimet.mpg.de) 

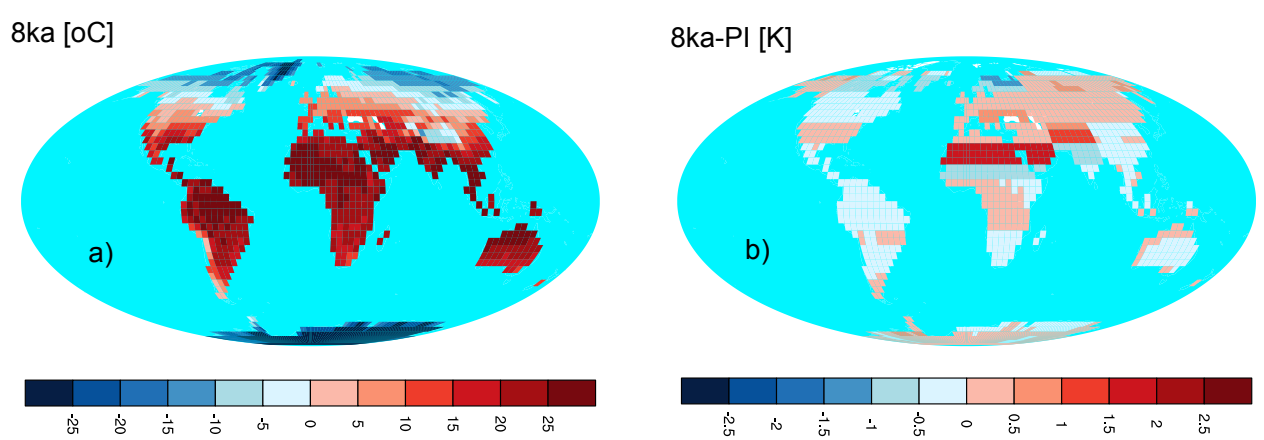

8ka [mm yr-1]

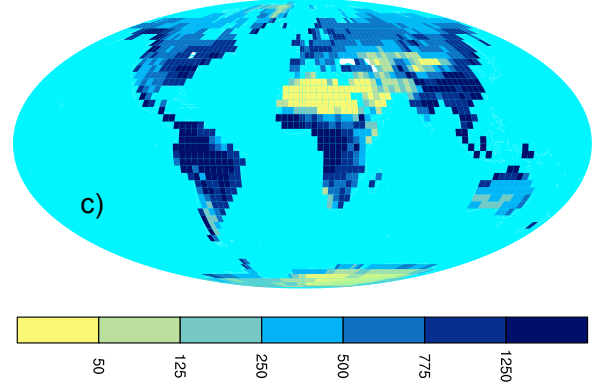

8ka-PI [mm yr-1]

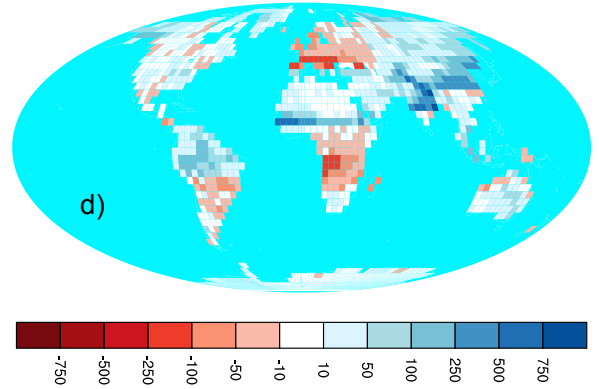

$8 \mathrm{ka}[]$

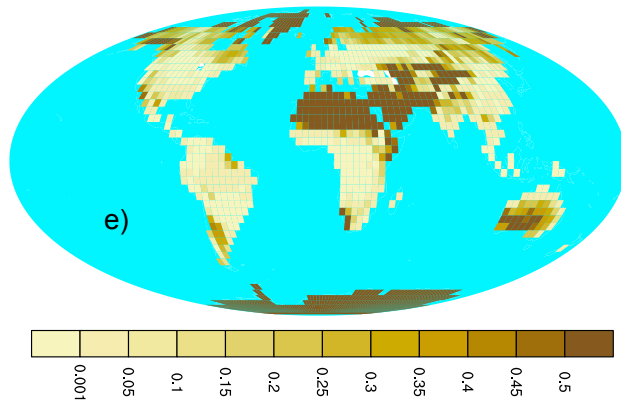

8ka-PI [ ]

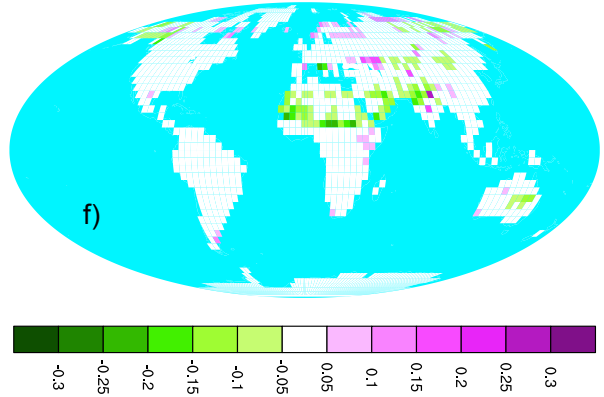

8ka-PI [ ]

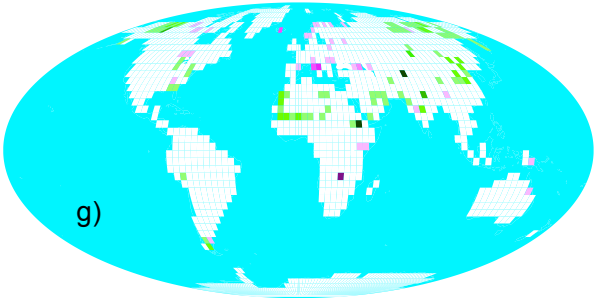

8ka-PI [ ]
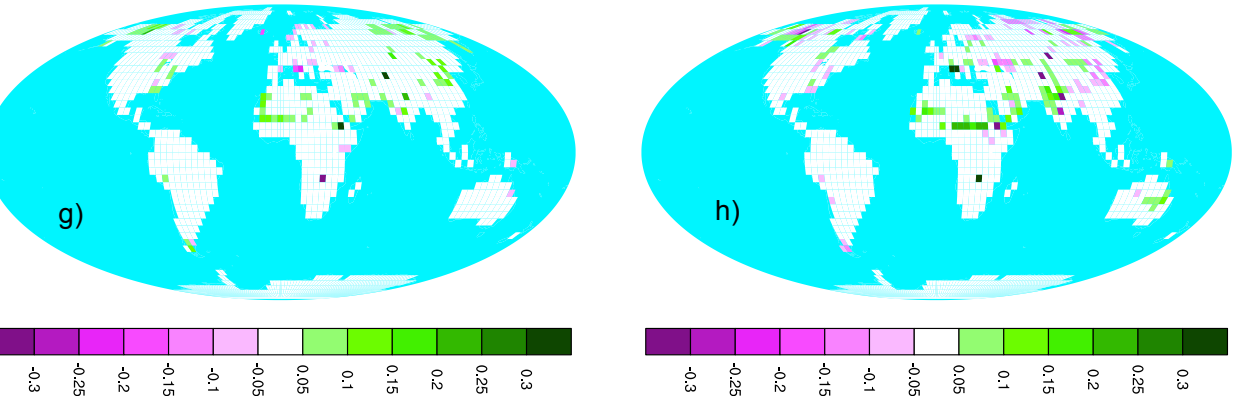

Fig. S1. Maps of climate and vegetation during the Holocene $(8 \mathrm{ka}=8000$ calyrs BP) and its differences to pre industrial climate (PI200 calyrs BP). Shown are $100 \mathrm{yr}$ averages for temperature $\left[{ }^{\circ} \mathrm{C}\right](\mathrm{a}+\mathrm{b})$, precipitation $\left[\mathrm{mm} \mathrm{yr}^{-1}\right](\mathrm{c}+\mathrm{d})$, desert fraction []$(\mathrm{e}, \mathrm{f})$, changes in forest [ ] (g) and grass (h) fraction []. 

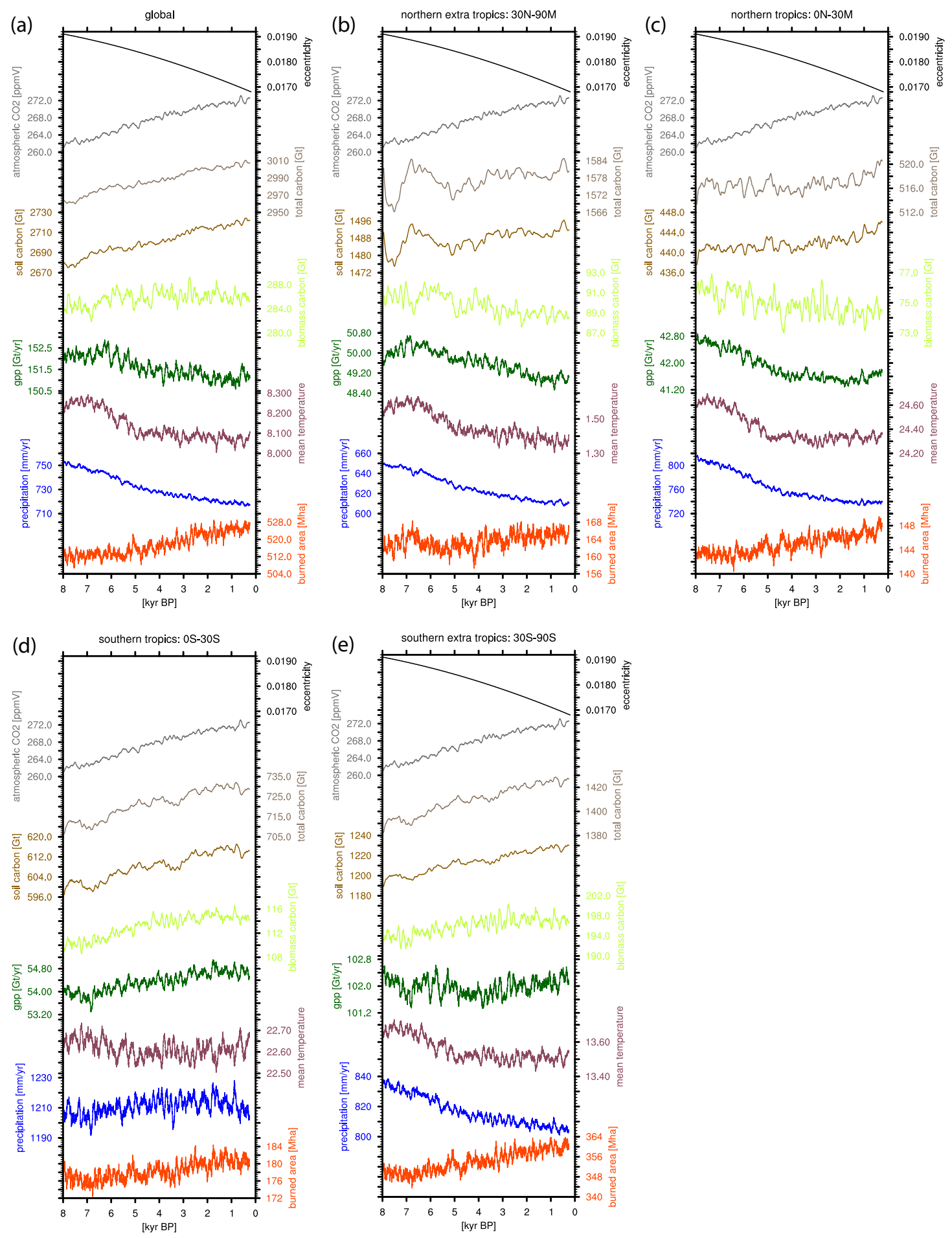

Fig. S2. Time series of hemispheric scale averaged model results over land. Shown are anomalies in burned area [Mha] (red), precipitation $\left[\mathrm{mmyr}^{-1}\right]$ (blue), yearly mean temperature $\left[{ }^{\circ} \mathrm{C}\right]$ (dark red), GPP [Gt yr $\left.{ }^{-1}\right]$ (dark green), biomass carbon [Gt] (light green), soil carbon [Gt] (brown), total carbon [Gt] (dark brown), atmospheric $\mathrm{CO}_{2}$ concentration [ppm] (gray), and eccentricity (black) for the global land area (a), northern and southern extra tropics $(b+e)$, as well as northern and southern tropics $(c+d)$. 

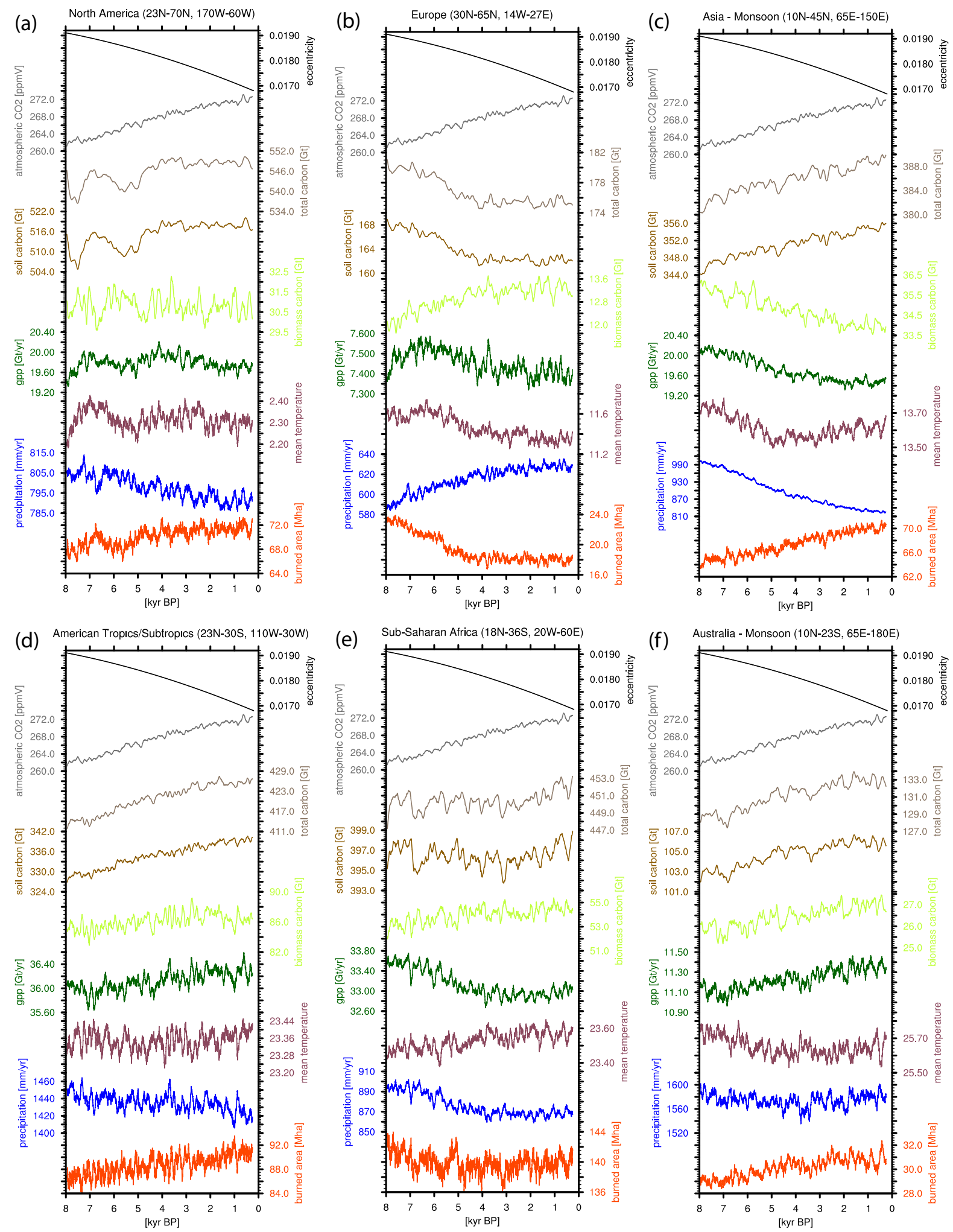

Fig. S3. Same as Fig. S2, but for the regional domains North America (a), Europe (b), Asia Monsoon (c), American Tropics (d), Sub-Saharan Africa (e), and Australia (f) given in Fig. ??. 
a) global

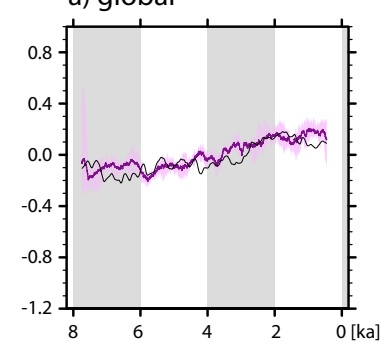

b) Northern hemisphere

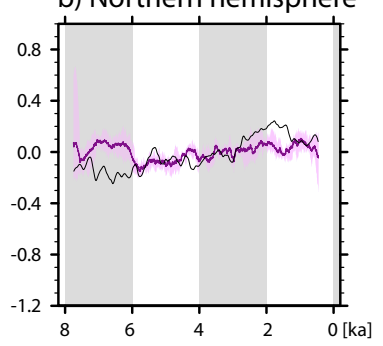

e) Southern hemisphere

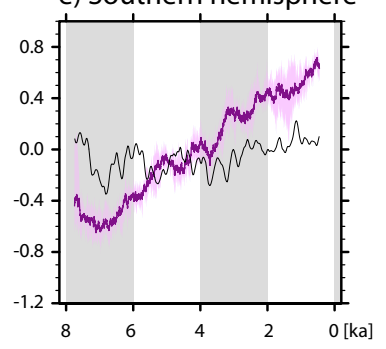

c) Northern extra-tropics

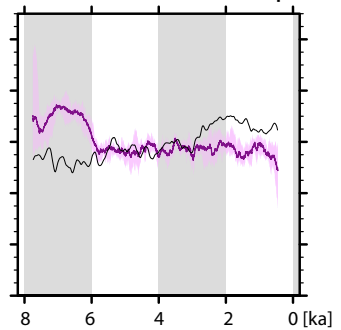

f) Southern tropics

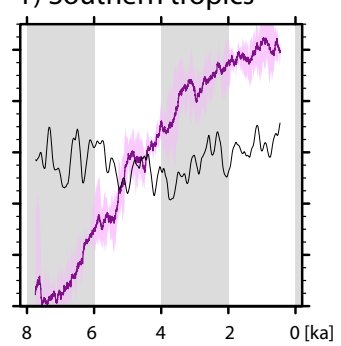

d) Northern tropics

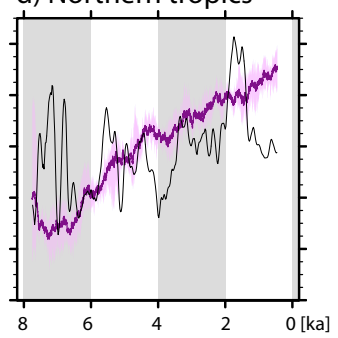

g) Southern extra-tropics

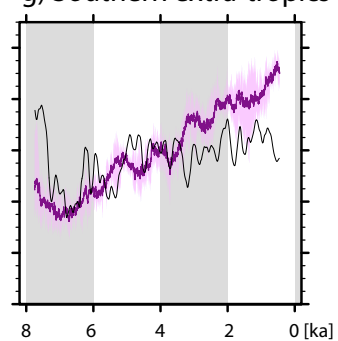

Fig. S4. Time series of global (a) and hemispheric scale (b-g) $Z$-score transformed and averaged burned area. Shown is the spread out of four ensemble members (pink) and the mean (red) plus the $Z$-scores out of charcoal reconstructions in black (median only). 
a) North America

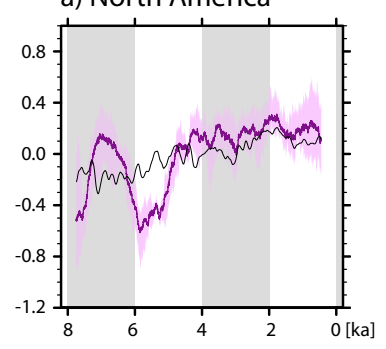

d) Central America Tropics

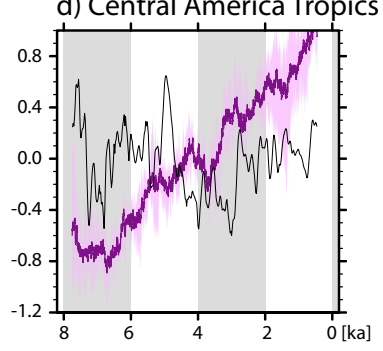

b) Europe

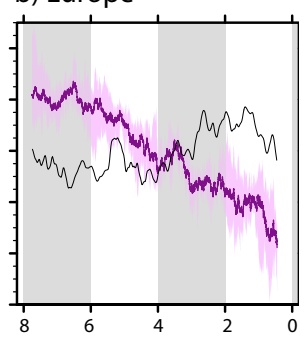

e) Africa

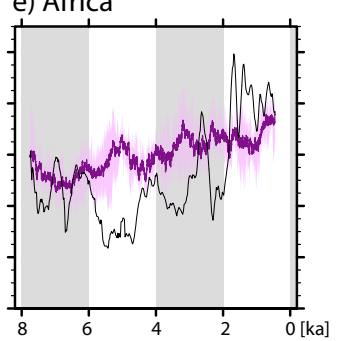

c) Asia monsoon

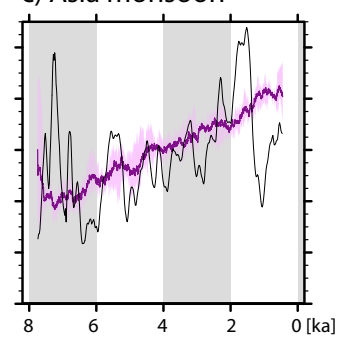

f) Australia monsoon

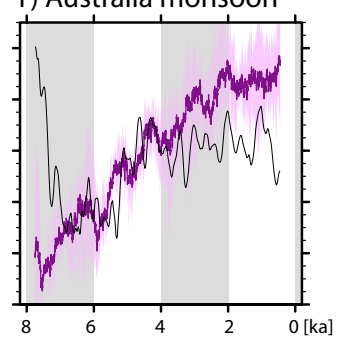

Fig. S5. Same as Fig. S4, but for regional domains given in Fig. ??. 
Table S1. Summary of all Spearman correlation coefficients $(\rho)$ and their significance level (p) for each region and time frame. Shown are the values for correlations based on $Z$-scores from reconstructed charcoal influxes (Z), untransformed and $Z$-score transformed model output of burned area $\left(\mathrm{F}, \mathrm{F}^{Z}\right)$ or carbon emissions $\left(\mathrm{C}, C^{Z}\right)$. Correlation coefficients that are negative or insignificant on a $5 \%$ significance level are in light gray. All correlations higher than 0.4 are bold and printed in blue $(\rho=0.4 \ldots 0.7)$, purple $(\rho=0.7 \ldots 0.8)$, or red $(\rho>0.8)$.

\begin{tabular}{|c|c|c|c|c|c|c|c|}
\hline \multirow[t]{2}{*}{ Area } & \multirow{2}{*}{$\begin{array}{l}\text { correlation } \\
\text { coefficient }\end{array}$} & \multicolumn{2}{|c|}{ 8ka-PI } & $\begin{array}{r}\text { Tin } \\
8 \mathrm{k}\end{array}$ & $\begin{array}{l}\text { frame } \\
4 \mathrm{ka}\end{array}$ & 4ka-PI & \\
\hline & & $\rho$ & $\mathrm{p}$ & $\rho$ & $\mathrm{p}$ & $\rho$ & $\mathrm{p}$ \\
\hline \multirow{4}{*}{ Global } & $\rho(\mathrm{Z}, \mathrm{F})$ & 0.18 & $<0.01$ & 0.22 & $<0.01$ & 0.04 & 0.63 \\
\hline & $\rho\left(\mathrm{Z}, \mathrm{F}^{Z}\right)$ & 0.73 & $<0.01$ & 0.49 & $<0.01$ & -0.02 & 0.79 \\
\hline & $\rho\left(\mathrm{Z}, \mathrm{C}^{Z}\right)$ & 0.77 & $<0.01$ & 0.48 & $<0.01$ & 0.20 & 0.01 \\
\hline & $\rho\left(\mathrm{F}^{Z}, \mathrm{C}^{Z}\right)$ & 0.98 & $<0.01$ & 0.99 & $<0.01$ & 0.91 & $<0.01$ \\
\hline \multirow{4}{*}{ Northern extra tropics } & $\rho(\mathrm{Z}, \mathrm{F})$ & 0.05 & 0.32 & 0.06 & 0.46 & -0.04 & 0.63 \\
\hline & $\rho\left(\mathrm{Z}, \mathrm{F}^{Z}\right)$ & -0.16 & 0.32 & -0.11 & 0.15 & -0.45 & $<0.01$ \\
\hline & $\rho\left(\mathrm{Z}, \mathrm{C}^{Z}\right)$ & -0.32 & $<0.01$ & -0.36 & $<0.01$ & -0.47 & $<0.01$ \\
\hline & $\rho\left(\mathrm{F}^{Z}, \mathrm{C}^{Z}\right)$ & 0.95 & $<0.01$ & 0.90 & $<0.01$ & 0.96 & $<0.01$ \\
\hline \multirow{4}{*}{ Northern tropics } & $\rho(\mathrm{Z}, \mathrm{F})$ & 0.13 & 0.01 & 0.12 & 0.11 & 0.11 & 0.14 \\
\hline & $\rho\left(\mathrm{Z}, \mathrm{F}^{Z}\right)$ & 0.42 & 0.01 & 0.15 & 0.04 & 0.19 & 0.01 \\
\hline & $\rho\left(Z, C^{Z}\right)$ & 0.42 & $<0.01$ & 0.21 & $<0.01$ & 0.13 & 0.09 \\
\hline & $\rho\left(\mathrm{F}^{Z}, \mathrm{C}^{Z}\right)$ & 0.99 & $<0.01$ & 0.96 & $<0.01$ & 0.98 & $<0.01$ \\
\hline \multirow{4}{*}{ Southern tropics } & $\rho(\mathrm{Z}, \mathrm{F})$ & 0.12 & 0.02 & 0.14 & 0.06 & 0.15 & 0.04 \\
\hline & $\rho\left(\mathrm{Z}, \mathrm{F}^{Z}\right)$ & 0.45 & 0.02 & 0.37 & $<0.01$ & 0.20 & 0.01 \\
\hline & $\rho\left(\mathrm{Z}, \mathrm{C}^{Z}\right)$ & 0.48 & $<0.01$ & 0.38 & $<0.01$ & 0.29 & $<0.01$ \\
\hline & $\rho\left(\mathrm{F}^{Z}, \mathrm{C}^{Z}\right)$ & 0.99 & $<0.01$ & 0.97 & $<0.01$ & 0.97 & $<0.01$ \\
\hline \multirow{4}{*}{$\begin{array}{l}\text { Southern } \\
\text { extra tropics }\end{array}$} & $\rho(\mathrm{Z}, \mathrm{F})$ & 0.02 & 0.76 & -0.02 & 0.82 & $<0.01$ & 0.97 \\
\hline & $\rho\left(\mathrm{Z}, \mathrm{F}^{Z}\right)$ & 0.24 & 0.76 & 0.66 & $<0.01$ & -0.26 & $<0.01$ \\
\hline & $\rho\left(Z, C^{Z}\right)$ & 0.22 & $<0.01$ & 0.63 & $<0.01$ & -0.32 & $<0.01$ \\
\hline & $\rho\left(\mathrm{F}^{Z}, \mathrm{C}^{Z}\right)$ & 0.99 & $<0.01$ & 0.96 & $<0.01$ & 0.98 & $<0.01$ \\
\hline \multirow{4}{*}{ North America } & $\rho(\mathrm{Z}, \mathrm{F})$ & 0.14 & 0.01 & 0.08 & 0.28 & -0.01 & 0.93 \\
\hline & $\rho\left(\mathrm{Z}, \mathrm{F}^{Z}\right)$ & 0.66 & 0.01 & -0.11 & 0.14 & 0.22 & $<0.01$ \\
\hline & $\rho\left(Z, C^{Z}\right)$ & 0.62 & $<0.01$ & -0.38 & $<0.01$ & 0.29 & $<0.01$ \\
\hline & $\rho\left(\mathrm{F}^{Z}, \mathrm{C}^{Z}\right)$ & 0.98 & $<0.01$ & 0.90 & $<0.01$ & 0.99 & $<0.01$ \\
\hline \multirow{4}{*}{ Europe } & $\rho(\mathrm{Z}, \mathrm{F})$ & -0.24 & $<0.01$ & -0.02 & 0.74 & -0.11 & 0.13 \\
\hline & $\rho\left(\mathrm{Z}, \mathrm{F}^{Z}\right)$ & -0.69 & $<0.01$ & -0.40 & $<0.01$ & -0.09 & 0.20 \\
\hline & $\rho\left(\mathrm{Z}, \mathrm{C}^{Z}\right)$ & -0.69 & $<0.01$ & -0.40 & $<0.01$ & -0.10 & 0.17 \\
\hline & $\rho\left(\mathrm{F}^{Z}, \mathrm{C}^{Z}\right)$ & 1.00 & $<0.01$ & 0.99 & $<0.01$ & 0.99 & $<0.01$ \\
\hline \multirow{4}{*}{$\begin{array}{l}\text { Central America } \\
\text { Tropics }\end{array}$} & $\rho(\mathrm{Z}, \mathrm{F})$ & 0.14 & 0.01 & 0.11 & 0.12 & 0.18 & 0.02 \\
\hline & $\rho\left(\mathrm{Z}, \mathrm{F}^{Z}\right)$ & -0.09 & 0.01 & 0.51 & $<0.01$ & -0.20 & 0.01 \\
\hline & $\rho\left(Z, C^{Z}\right)$ & -0.04 & 0.47 & 0.67 & $<0.01$ & -0.14 & 0.07 \\
\hline & $\rho\left(\mathrm{F}^{Z}, \mathrm{C}^{Z}\right)$ & 0.99 & $<0.01$ & 0.95 & $<0.01$ & 0.98 & $<0.01$ \\
\hline \multirow{4}{*}{ Africa } & $\rho(\mathrm{Z}, \mathrm{F})$ & 0.03 & 0.59 & 0.14 & 0.05 & -0.08 & 0.30 \\
\hline & $\rho\left(\mathrm{Z}, \mathrm{F}^{Z}\right)$ & 0.32 & 0.59 & 0.23 & $<0.01$ & -0.24 & $<0.01$ \\
\hline & $\rho\left(\mathrm{Z}, \mathrm{C}^{Z}\right)$ & 0.46 & $<0.01$ & 0.61 & $<0.01$ & -0.46 & $<0.01$ \\
\hline & $\rho\left(\mathrm{F}^{Z}, \mathrm{C}^{Z}\right)$ & 0.86 & $<0.01$ & 0.84 & $<0.01$ & 0.69 & $<0.01$ \\
\hline \multirow{4}{*}{ Australian Monsoon } & $\rho(\mathrm{Z}, \mathrm{F})$ & 0.02 & 0.66 & -0.07 & 0.33 & -0.02 & 0.76 \\
\hline & $\rho\left(\mathrm{Z}, \mathrm{F}^{Z}\right)$ & 0.39 & 0.66 & 0.03 & 0.66 & 0.08 & 0.30 \\
\hline & $\rho\left(\mathrm{Z}, \mathrm{C}^{Z}\right)$ & 0.42 & $<0.01$ & 0.16 & 0.03 & 0.11 & 0.15 \\
\hline & $\rho\left(\mathrm{F}^{Z}, \mathrm{C}^{Z}\right)$ & 0.98 & $<0.01$ & 0.95 & $<0.01$ & 0.94 & $<0.01$ \\
\hline \multirow{4}{*}{ Asia monsoon } & $\rho(\mathrm{Z}, \mathrm{F})$ & 0.18 & $<0.01$ & 0.09 & 0.23 & 0.08 & 0.31 \\
\hline & $\rho\left(\mathrm{Z}, \mathrm{F}^{Z}\right)$ & 0.48 & $<0.01$ & 0.09 & 0.22 & 0.23 & $<0.01$ \\
\hline & $\rho\left(\mathrm{Z}, \mathrm{C}^{Z}\right)$ & 0.45 & $<0.01$ & -0.07 & 0.35 & 0.21 & 0.01 \\
\hline & $\rho\left(\mathrm{F}^{Z}, \mathrm{C}^{Z}\right)$ & 0.99 & $<0.01$ & 0.95 & $<0.01$ & 0.97 & $<0.01$ \\
\hline
\end{tabular}

